\section{Multiple pathways inhibit NHEJ at telomeres}

\author{
Stéphane Marcand, 1,5 Benjamin Pardo, ${ }^{1,3}$ \\ Ariane Gratias, ${ }^{1,4}$ Sabrina Cahun, ${ }^{1}$ and \\ Isabelle Callebaut ${ }^{2}$ \\ ${ }^{1}$ Centre National de la Recherche Scientifque (CNRS) UMR \\ 217, Institut de Radiobiologie Cellulaire et Moléculaire \\ (IRCM), CEA/Fontenay, 92265 Fontenay-aux-roses cedex, \\ France; ${ }^{2}$ CNRS UMR 7590, Universités \\ Paris 6 et Paris 7, 75015 Paris, France
}

The nonhomologous end-joining (NHEJ) repair pathway is inhibited at telomeres, preventing chromosome fusion. In budding yeast Saccharomyces cerevisiae, the Rap1 protein directly binds the telomere sequences and is required for NHEJ inhibition. Here we show that the Rap1 C-terminal domain establishes two parallel inhibitory pathways through the proteins Rif2 and Sir4. In addition, the central domain of Rap1 inhibits NHEJ independently of Rif2 and Sir4. Thus, Rap1 establishes several independent pathways to prevent telomere fusions. We discuss a possible mechanism that would explain Rif2 multifunctionality at telomeres and the recent evolutionary origin of Rif 2 from an origin recognition complex (ORC) subunit.

Supplemental material is available at http://www.genesdev.org.

Received August 30, 2007; revised version accepted February 29, 2008.

Nonhomologous end-joining (NHEJ) inhibition at telomeres prevents fusions between two telomeres and between a telomere and a double-stranded broken end (DuBois et al. 2002; Chan and Blackburn 2003; Mieczkowski et al. 2003; Riha et al. 2006). In fission yeast Schizosaccharomyces pombe, the Tazl protein binds telomeric DNA and recruits the Rap1 protein. Both Taz1 and Rap1 are required for NHEJ inhibition (Ferreira and Cooper 2001; Miller et al. 2005). In mammals, TRF1 and TRF2 are the two orthologs of Taz1, and RAP1 is recruited to the telomeres by TRF2 (Li et al. 2000). In vivo, TRF2 is essential for NHEJ inhibition (van Steensel et al. 1998; Celli and de Lange 2005; Celli et al. 2006). In vitro, it has been found that NHEJ inhibition by telomeric repeats requires TRF2 and RAP1 (Bae and Baumann 2007). In Saccharomyces cerevisiae, there is no ortholog of Taz1/ TRF1/TRF2. Rap1 directly binds the telomere sequences, and its loss causes telomere fusions dependent on all the factors known to be required for NHEJ (Pardo and Marcand 2005; Pardo et al. 2006).

[Keywords: Genome stability; NHEJ; Rap1; Rif2; Sir4; telomere] Present addresses: ${ }^{3}$ Department of Molecular Biology, Centro Andaluz de Biología Molecular y Medicina Regenerativa (CABIMER), Avenida America Vespucio S/N, 41092 Sevilla, Spain; ${ }^{4}$ Université Paris 11, IBP, Bat 630, 91160 Orsay, France.

${ }^{5}$ Corresponding author:

E-MAIL stephane.marcand@cea.fr; FAX 33-1-46549180.

Article is online at http://www.genesdev.org/cgi/doi/10.1101/gad.455108.
Rap1 is a modular protein with three conserved domains: a BRCT domain of unknown function in the $\mathrm{N}$ terminal region, a centrally located DNA-binding domain with two Myb-like folds, and an independent Cterminal domain called RCT (Rap1 C-terminal) (Fig. 1A; Li et al. 2000; Dreesen et al. 2007). In budding yeast, Rap 1 also binds to $\sim 5 \%$ of the promoters and is required for viability. The BRCT and RCT domains are dispensable (Kyrion et al. 1992; Moretti et al. 1994). The RCT is required for the negative feedback loop that represses telomere elongation by telomerase (Kyrion et al. 1992; Marcand et al. 1997) and for the establishment of a silent chromatin near telomeres (Kyrion et al. 1993; Buck and Shore 1995). It interacts with the proteins Rif1, Rif2, Sir3, and Sir4. Rif1 and Rif2 are required for the inhibition of telomere elongation (Wotton and Shore 1997; Levy and Blackburn 2004; Teixeira et al. 2004). Sir3 and Sir4 establish transcriptional silencing, which also requires Sir2, a conserved histone deacetylase. In this work, we ask which Rapl domains and interacting factors are involved in NHEJ inhibition at telomeres.

\section{Results and Discussion}

\section{Rap1 C-terminal domain is required for NHEJ inhibition at telomeres}

In $S$. cerevisiae, the $\mathrm{TG}_{1-3}$ telomere repeats display a variability that to some degree disrupts the palindrome formed by a telomere-telomere fusion, allowing the detection of a fraction of the fusions directly by PCR (Fig. 1B; Mieczkowski et al. 2003; Pardo and Marcand 2005). Initially, we looked for fusions in a rap1- $\Delta C$ mutant lacking the RCT $(\Delta 670-807)$, but failed to detect them (Fig. 1C; data not shown). In rap1- $\Delta C$ cells, telomeres are elongated over several kilobases (Kyrion et al. 1992), and fusions between long telomeres may not be prone to amplification. In tel1 and tel1 rap1- $\Delta C$ cells, telomeres are short (Craven and Petes 1999), at a steady mean length of $\sim 160$ and $\sim 200$ base pairs (bp), respectively, in the strains used here. On its own, the absence of Tell does not influence the frequency of fusions caused by full-length Rap1 loss (Pardo and Marcand 2005) and only moderately changes the frequency of rearrangements between two double-strand breaks and between a double-strand break and a telomere (DuBois et al. 2002; Chan and Blackburn 2003; Pennaneach and Kolodner 2004; Lee and Lee 2007), suggesting that we could take advantage of the tel1- $\Delta$ mutant to facilitate the detection of fusions by PCR. As shown in Figure 1C, telomere fusions are amplified by PCR in the tel1 rap1- $\Delta C$ double mutant. Telomere length distribution being heterogeneous, telomere fusions are detected as a smearing PCR signal. The mean length of the PCR products suggests fusions with $\sim 350$ bp of telomeric repeats, a length consistent with fusions between telomeres close to the mean telomere length in this strain. Fusions remain undetected in wild-type cells, in a tel1 single mutant, and in tel1 rap1- $\Delta C$ cells lacking the NHEJ factor Lif1. This result suggests that the Rap1 C-terminal domain is required for NHEJ inhibition at telomeres, at least in a context where the telomeres remain short. 
Marcand et al.

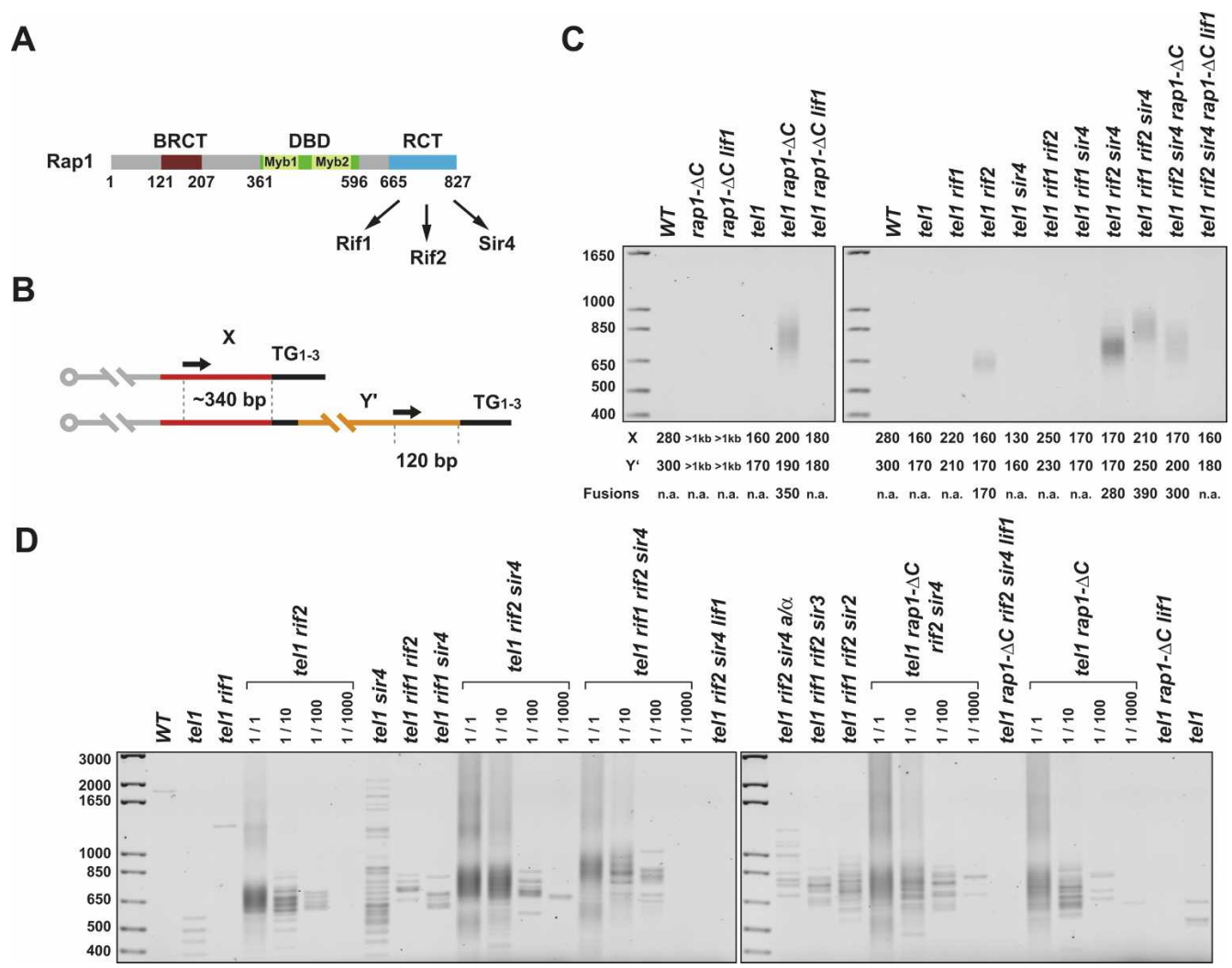

Figure 1. NHEJ inhibition at telomeres requires the Rap1 C-terminal domain, Rif2, and Sir4. $(A)$ Schematic representation of S. cerevisiae Rap1. (B) Schematic representation of X and $\mathrm{Y}^{\prime}$ telomeres in $S$. cerevisiae and relative positions of the primers used for PCR amplification. $(C)$ Fusions amplified after 26 cycles. The mean lengths of the $\mathrm{X}$ and $\mathrm{Y}^{\prime}$ telomeres and of the amplified fusions are indicated in base pairs below each lane. $(D)$ Fusions amplified after 33 cycles.

\section{Rif2 and Sir4 are required for NHEJ inhibition at telomeres}

We then asked whether NHEJ inhibition requires factors known to interact with the RCT. As shown in Figure 1C, fusions are detected in tel1 rif2 cells, indicating that the loss of Rif 2 is sufficient to cause fusions in a tel1- $\Delta$ context. Their average length is about half the expected length from the mean telomere length in this strain, suggesting that only the shortest telomeres are exposed to NHEJ in tel1 rif2 cells. In tel1 rif2 sir4 cells, fusions are also detected, but instead display an average length close to the one expected from the mean telomere length in this strain. This shows that Sir4 is required to avoid fusions between telomeres of average length in tel1 rif2 cells. Interestingly, fusions detected in tel1 rif2 cells are lost in tel1 rif1 rif2 cells. This may be due to the slight telomere elongation caused by Rif1 loss, eliminating the short telomeres exposed to NHEJ. The loss of Rif1 may also improve the recruitment of Sir4 at telomeres, as shown previously (Buck and Shore 1995; Mishra and Shore 1999).

Fusions are detected in tel1 rif1 rif2 sir4 cells but not in tel1 rif1 rif2 cells, although they display telomeres of similar length (Fig. 1C). This shows that Sir4 is needed to inhibit fusions in tel1 rif1 rif2 cells. Similarly, fusions are detected in tel1 rif1 rif2 sir4 cells but not in tel1 rif1 sir4, indicating that Rif2 is required to inhibit fusions in tel1 rif1 sir4 cells. Finally, tel1 rif2 sir4 rap1- $\Delta C$ cells display fusions at similar levels and lengths to tel1 rif2 sir4 and tel1 rap1- $\Delta C$ cells, suggesting that the loss of NHEJ inhibition in cells lacking the Rapl C-terminal domain is caused by the loss of Rif2 and Sir4 at telomeres. No fusion could be detected in tel1 rif2 sir4 rap1$\Delta C$ cells lacking the NHEJ factor Lif1. Together, these initial observations indicate that Rif2 and Sir4 independently inhibit NHEJ but not Rif1.

The signals shown in Figure 1C were obtained after 26 PCR cycles. With 33 cycles, the PCR uncovers fusions occurring at a lower frequency in some strains. Thus, rare fusions are detected in tel1 cells, tel1 sir4 cells, tel1 rif1 rif2 cells, and tel1 rif1 sir4 cells (Fig. 1D; Supplemental Fig. 1). In wild-type cells, tel1 rif1 cells, and cells lacking Lif1, products whose length would be consistent with telomere fusions remain undetected. When fusions are frequent and saturate the PCR, the products spread within the gel. Reannealing between different PCR products with unmatched telomeric sequences could explain this pattern. For those strains displaying a high fusion frequency, the genomic DNA was serially diluted within the genomic DNA of a wild-type strain, keeping the amount of DNA template constant. As the fusions become less abundant, the product pattern changes from a smear to a set of discrete bands, probably because a few fusions are preferentially amplified.

Whereas a sir4 deletion increases the frequency of fusions in a tel1 rif1 rif2 mutant by about two orders of magnitude, a sir2 or sir3 deletion causes only a slight increase (Fig. 1D). This indicates a unique role for Sir4 in NHEJ inhibition that does not require the assembly of a 


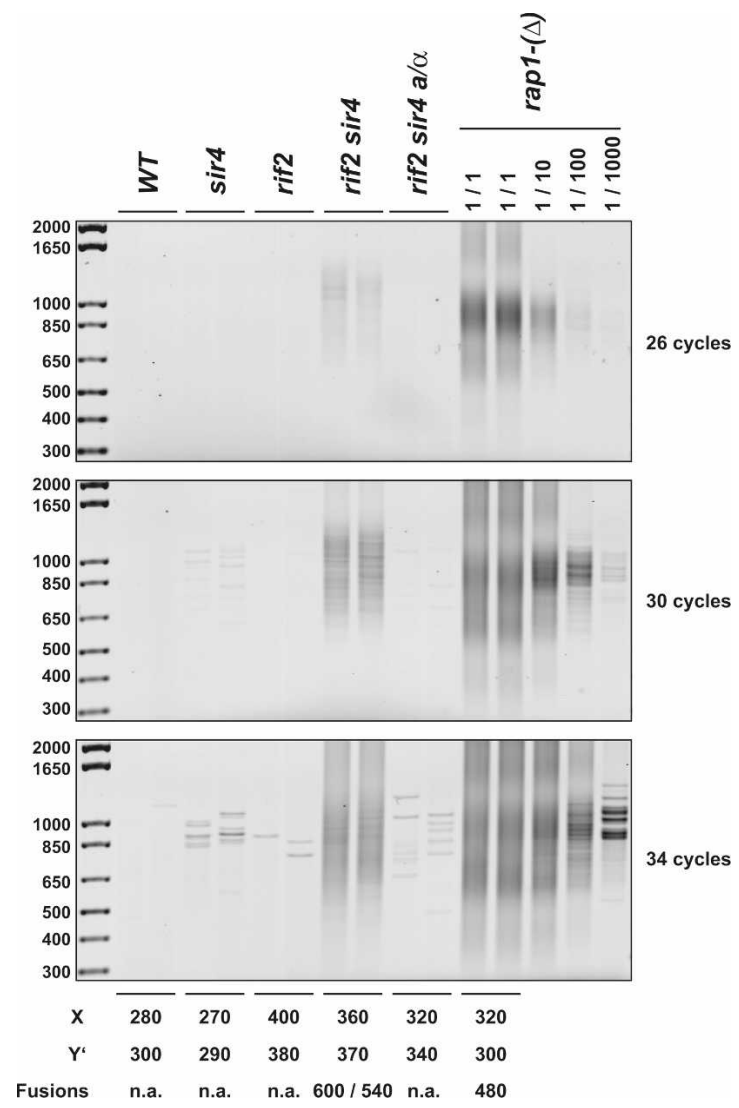

Figure 2. Amplification of telomere-telomere fusions in TEL1 cells. For each strain, PCRs from two independent samples are displayed. DNA was diluted with DNA from the wild-type strain.

silent chromatin. In a/ $\alpha$ cells, NHEJ is repressed globally (Astrom et al. 1999). Thus, in tel1 rif2 sir4 a/ $\alpha$ cells, the frequency of fusions is reduced, as expected.

The requirement of Rif2 and Sir4 for NHEJ inhibition is apparent in tel1- $\Delta$ strains in which telomeres are shorter than in wild-type cells. We then asked whether this requirement is detectable in TEL1 cells. As shown in Figure 2, fusions are amplified after a limited number of cycles in rif2 sir4 cells but not in rif2 and sir4 single mutant cells nor in rif2 sir 4 a/ $\alpha$ cells. A very low frequency of telomere-telomere fusions in rif2 cells is consistent with a previous report showing that fusions between telomeres and a double-strand break remain rare in rif2 cells (DuBois et al. 2002).

\section{NHEJ inhibition by Rap1 in the absence of Rif2 and Sir4}

To compare the frequency of fusions between cells lacking Rif2 and Sir4 and cells losing Rap1, we used a previously described conditional allele of $R A P 1$, a double shut-off degron called rap1-( $\Delta$ ) (Pardo and Marcand 2005). This allele causes Rap1 levels to drop in cells exiting exponential growth. As shown in Figure 2, fusions are much more frequently amplified in rap1-( $(\Delta)$ cells than in rif2 sir4 cells, suggesting that NHEJ remains partially inhibited in cells lacking Rif2 and Sir4. However, telomeres are longer in rif2 sir4 cells than in rap1-( $(\Delta)$ cells and, remarkably, the mean length of the amplified fusions in rif2 sir4 cells changes with the number of PCR cycles from $\sim 600$ bp at 26 cycles to $\sim 540$ at 30 cycles. The preferential amplification of shorter products during the PCR makes the comparison between two strains with different telomere lengths less convincing.

To address this difficulty, the frequencies of fusions in cells lacking Rif2 and Sir4 and in cells losing Rap1 were compared in a tel1- $\Delta$ context. As shown in Figure 3, the highest level of fusions is observed in tel1 rif2 sir4 rap1$(\Delta)$ cells in which telomeres are also the longest $(-240$ bp). PCR performed on serial dilutions of their genomic DNA allows an estimation of the relative fusion frequencies between the tested tel1 strains. Fusions are at least 30 times more frequent in tel1 rif2 sir4 rap1-( $\Delta)$ cells than in tel1 rif2 sir4 cells or in tel1 rif2 sir4 rap1- $(\Delta)$ cells complemented with a wild-type copy of RAP1 [tel1 rif2 sir4 rap1- $(\Delta)+R A P 1]$. This indicates that Rapl still inhibits NHEJ in the absence of Rif2 and Sir4. tel1 rif2 sir4 rap1-( $\Delta$ ) cells display a significantly higher level of fusions than tel1 rap1- $(\Delta)$ cells (Fig. 3, 20 cycles), indicating that the rap1- $(\Delta)$ allele is not a complete loss-of-function mutant for the Rif2 and Sir4 pathways. Fusions are at least 10 times more frequent in tel1 rif2 sir4 cells than in tel1 rif2 cells or in tel1 sir4 cells, confirming the synergistic interaction between Rif2 and Sir4 to inhibit NHEJ.

Since tel1 rif2 sir4 rap1- $\Delta C$ cells do not display more fusions than tel1 rif2 sir4 cells (Fig. 1), the loss of the Rap1 C-terminal domain does not explain the increased level of fusions caused by full-length Rap1 loss in a tel1 rif2 sir4 mutant context. Hence, we used a rap1- $\Delta N$ allele encoding a protein truncated for its nonessential $\mathrm{N}$-terminal part $(\Delta 2-228)$ to determine whether the Rap1

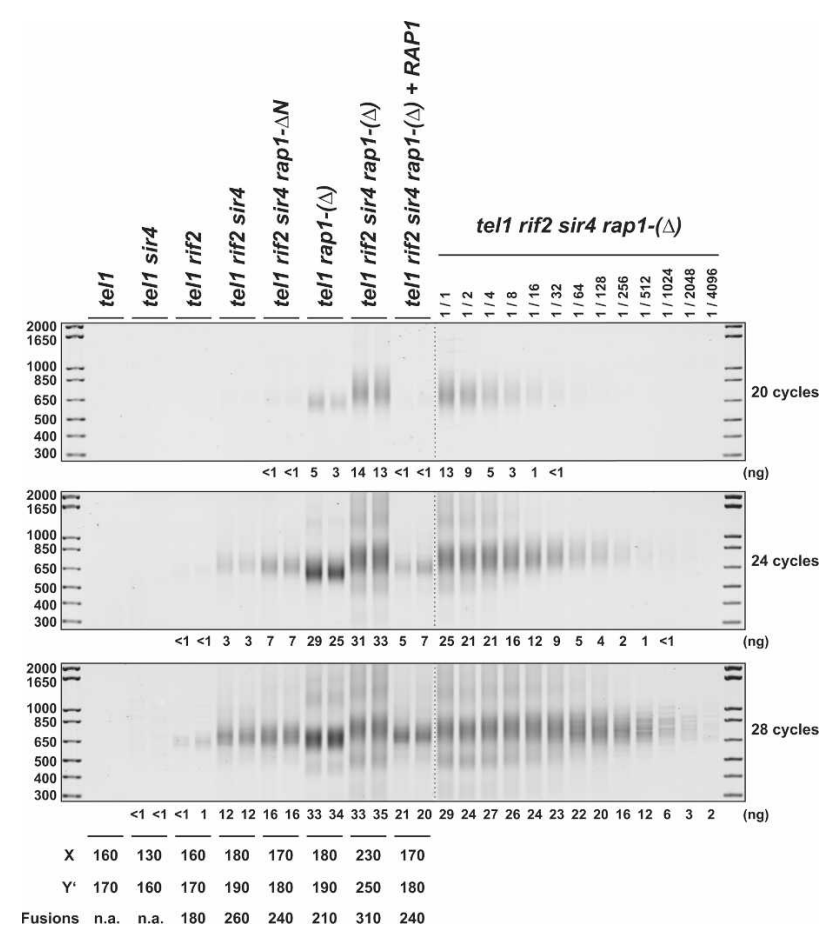

Figure 3. NHEJ inhibition by Rap 1 in the absence of Rif2 and Sir4 For each strain, PCRs from two independent samples are displayed. The amount of amplified product is indicated in nanograms below each visible smear. DNA were diluted with DNA from the wild-type strain. 
BRCT domain could be involved in NHEJ inhibition. As shown in Figure 3, fusions in tel1 rif2 sir4 rap1- $\Delta N$ cells remain $\sim 30$ times less frequent than tel1 rif2 sir4 rap1$(\Delta)$ cells. This indicates that NHEJ inhibition in the absence of Rif2 and Sir4 does not entail the Rap1 N-terminal domain. Together, these results show that the part of Rap1 essential for viability, including the DNA-binding domain, establishes NHEJ inhibition through a pathway independent of Rif2 and Sir4.

\section{NHEJ inhibition in cis at double-strand breaks}

We asked whether NHEJ inhibition could also be established at another position in the genome. To test this, two I-SceI sites were inserted in opposite orientation on each side of the URA3 gene on chromosome $\mathrm{V}$ as displayed in Figure 4A. The sequence encoding the nuclease was inserted at a different locus and placed under the control of a galactose-inducible promoter. Cells are haploid and cannot survive the loss of the broken chromosome. Simple rejoining by NHEJ recreates the I-SceI sites. Upon continuous expression of the endonuclease on galactose, only $\sim 0.4 \%$ of the cells form a colony. These cells survive by mutating the cleavage sites. In

A

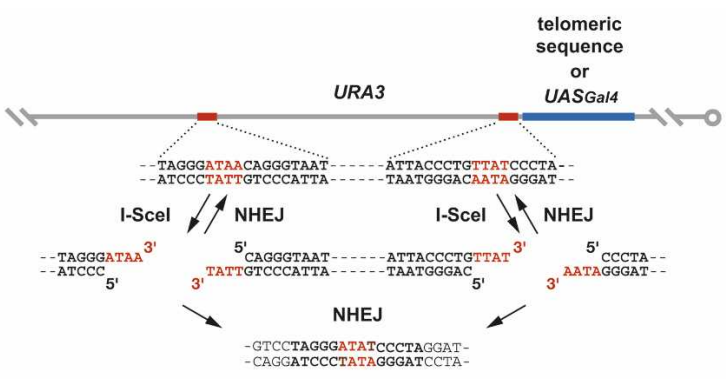

B

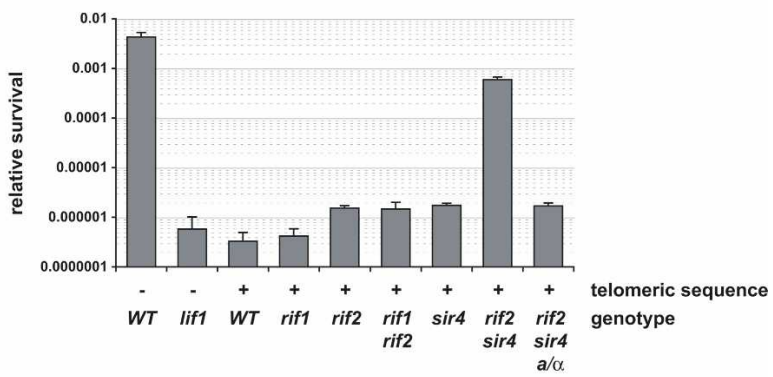

C

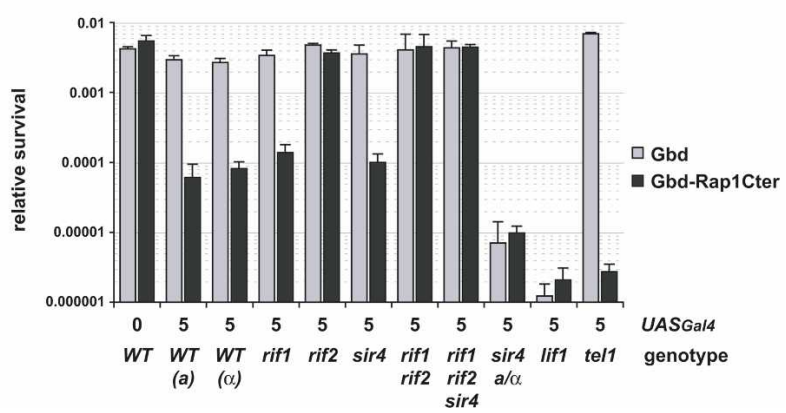

Figure 4. NHEJ inhibition at double-strand breaks. $(A)$ Schematic representation of the NHEJ assay. (B) NHEJ inhibition by internal telomeric repeats. $(C)$ NHEJ inhibition by ectopic targeting of Rap1 $\mathrm{C}$-terminal domain. The mean relative survival on galactose versus glucose were observed in three experiments. most of the survivors, the URA3 gene is lost and the two distal ATAA 3' overhangs are fused to form an -ATATjoint (data not shown). This event requires Lif1 and the DNA polymerase Pol4 (data not shown).

First, we asked whether a telomeric sequence adjacent to the break could inhibit NHEJ. Two-hundred-fifty-six base pairs of telomeric repeats were inserted in the vicinity of one of the I-SceI sites. The distance between the $5^{\prime}$ end generated by I-SceI and the edge of the telomeric repeats is $12 \mathrm{bp}$. The TG strand runs $5^{\prime}$ to $3^{\prime}$ toward the end. As shown in Figure $4 \mathrm{~B}$, the presence of the telomeric repeats reduced survival on galactose to the frequency observed in cells lacking Lif1. Thus, an internal telomeric sequence near a break inhibits a rearrangement by NHEJ. In cells lacking Rif2 or Sir4 alone, the frequency of survival is slightly increased by about fourfold. In cells lacking both Rif2 and Sir4, the frequency of survival is increased by $\sim 300$-fold compared with the single mutants. This indicates that Rif2 and Sir4 synergistically inhibit NHEJ in this assay. In rif2 sir 4 a/ $\alpha$ cells, NHEJ is globally repressed in trans and survival is reduced to a low frequency. The loss of Rif1 does not influence survival on galactose. Survival in a rif2 sir4 double mutant remains about sevenfold lower than in the absence of the telomeric sequence, suggesting that rearrangements by NHEJ are still partially inhibited in this context.

Next, we asked whether NHEJ inhibition could be established independently of the telomeric repeats. Five $U A S_{\text {Gal4 }}$-binding sites were inserted in the vicinity of one of the I-SceI sites replacing the internal telomeric sequence. The distance between the $5^{\prime}$ end generated by I-SceI and the edge of the first Gal4-binding site is $23 \mathrm{bp}$. In cells expressing the Gal4 DNA-binding domain alone (Gbd) and the native Gal4 protein, the presence of the $U A S_{G a 14}$ sites does not influence survival on galactose (Fig. 4C). To target the Rap1 C-terminal domain, we used a chimera fusing the Gal4 DNA-binding domain and the Rap1 C-terminal domain (Gbd-Rap1Cter; amino acids 653-827). This hybrid establishes transcriptional silencing and participates in the negative feedback loop on telomere elongation when targeted to the internal edge of a telomere (Buck and Shore 1995; Marcand et al. 1997).

In the NHEJ assay described here, Gbd-Rap1Cter reduces survival on galactose by $\sim 50$-fold in cells with $U A S_{\text {Gal4 }}$ sites adjacent to the break, and does not influence survival in cells without $U A S_{\text {Gal4 }}$ sites (Fig. 4C). Thus, targeting the Rap1 C-terminal domain is sufficient to inhibit a rearrangement by NHEJ at an ectopic position. This inhibition is not complete compared with a lif1- $\Delta$ mutant. Remarkably, inhibition is strengthened in cells lacking Tel1. In cells lacking Rif2, Gbd-Rap1Cter loses its ability to reduce the frequency of rearrangements. In cells lacking only Rif1 or Sir4, Gbd-Rap1Cter retains its repressive effect. In sir 4 a $/ \alpha$ cells, GbdRap1Cter does not influence the residual frequency of survival. The requirement for Rif2 but not Sir4 in this assay indicates that NHEJ inhibition by Gbd-Rap1Cter acts through the Rif2 pathway established by Rap1.

\section{Possible mechanisms of inhibition}

We observed that in budding yeast Rap1 establishes several parallel pathways to inhibit NHEJ at telomeres. The coexistence of independent inhibitory pathways ensures 
A

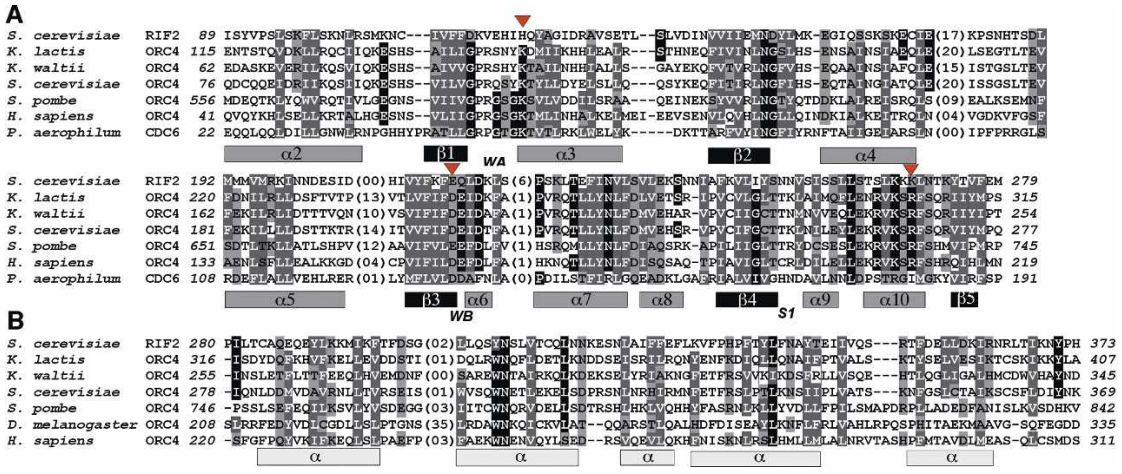

Figure 5. Alignment of the Rif2 and Orc4 sequences. (A) The first domain (domain I) of the $\mathrm{AAA}^{+}$module. The positions of the observed secondary structures of Pyrobaculum aerophilum Cdc6 (Liu et al. 2000) are shown at the bottom, together with those of AAA ${ }^{+}$motifs. (WA) Walker $\mathrm{A}_{;}$(WB) Walker $\mathrm{B}$; (S1) sensor 1. Identities are shown white on a black background, and similarities are shaded gray (dark gray for hydrophobic residues, light gray for other similarities). Positions of the Walker A lysine, Walker B aspartate, and S. cerevisiae Orc4 Arg 267 are indicated by red arrowheads. (B) The second domain (domain II) of the $\mathrm{AAA}^{+}$module. Only the sequences of Rif 2 and Orc4 are aligned, as the alignment cannot be extended in an accurate way to Cdc6. However, secondary structure prediction made on the basis of this alignment using PHD (Rost et al. 2004) indicated an all- $\alpha$ fold, with four main helices, reminiscent of the domain II structure observed in Cdc6. Note that in Cdc6, the domain II is discontinuous, with a short $\mathrm{N}$-terminal fragment located upstream of domain I. Such an $\mathrm{N}$-terminal extension might be found in the Rif2 and Orc4 sequences.

that fusions do not occur unless the telomeres become extremely short (Chan and Blackburn 2003; Mieczkowski et al. 2003). This buffering sees to it that fusions never form when telomeres deviate slightly from the range of lengths found in wild-type cells. In humans, short tracts of telomeric DNA are also sufficient to inhibit NHEJ in vitro (Bae and Baumann 2007).

NHEJ inhibition by an ectopic targeting of the Rap1 C-terminal domain near a double-strand break reveals that the inhibitory pathway that requires Rif2 can act without telomeric DNA. This suggests that Rif2 acts on protein complexes and not through a telomere-specific DNA structure. It also reveals that NHEJ inhibition by Rif2 is a standard cis effect in which the recruitment of a factor locally inhibits a molecular function. Rif2 inhibits not only NHEJ but also telomerase-mediated telomere elongation (Wotton and Shore 1997; Teixeira et al. 2004) and a form of homologous recombination between telomeres (Teng et al. 2000). A unique common requirement for these three inhibited processes is the Mre11-Rad50-Xrs2 complex. Thus, the simplest hypothesis is that the Rif2 pathway inhibits locally the Mre11-Rad50-Xrs2 complex. This single molecular activity for Rif2 would explain its multifunctionality. Wild-type-length telomeres are refractory to Mre11Rad50-Xrs2 binding (Negrini et al. 2007; Viscardi et al. 2007), suggesting that Rif2 may inhibit the recruitment of the complex.

Sir4 inhibits NHEJ through an unknown mechanism. Interestingly, Sir4 interacts at telomeres with the Yku80 subunit of KU (Ribes-Zamora et al. 2007). This interaction might be important for NHEJ inhibition. The central domain of Rap1 inhibits NHEJ independently of Rif2 and Sir4. Rap1 interaction with DNA might directly outcompete the stable recruitment of the NHEJ machinery or act through a DNA secondary structure similar to the one proposed for telomeres in higher eukaryotes (Griffith et al. 1999; Amiard et al. 2007).

\section{Evolutionary origin of Rif2}

Rapl is required for NHEJ inhibition in fission yeast (Miller et al. 2005) and in vitro with human cell extracts (Bae and Baumann 2007), suggesting that the pathways observed in budding yeast might be conserved in evolution. An ortholog of Sir4 in higher eukaryotes remains to be identified. Remarkably, RIF2 is syntenic to ORC4, indicating that the two genes diverged from a common ancestor after the last whole-genome duplication of the $S$. cerevisiae lineage (Scannell et al. 2007). Despite this synteny information, the similarity between the Rif 2 and Orc4 sequences can be detected only using very sensitive methods of sequence analysis (Fig. 5; Supplemental Fig. 2). Like Orc4, Rif2 possesses two domains typical of $\mathrm{AAA}^{+}$modules but is distinct from Orc4, since an additional C-terminal domain, adopting a winged-helix fold in Cdc6, is absent. The Walker A and Walker B motifs are highly degenerated in Rif2: The Walker A lysine is substituted by a histidine, while the Walker B aspartate, which is located at the end of a $\beta$-strand, is substituted by a glutamic acid. Arg 267 of $S$. cerevisiae Orc4, essential for origin recognition complex (ORC) ATPase activity (Bowers et al. 2004), is not conserved in Rif2. This suggests that, although it shares the same overall $\mathrm{AAA}^{+}$ fold as Orc4, Rif2 probably lacks ATPase activity.

The recent origin of Rif2 suggests two scenarios. Rif2 may have established a new pathway at telomeres; for instance, to compensate for the weakening of another pathway. Alternatively, the duplication of the gene may have allowed a separation of functions and a specialization of Rif2 at telomeres. If the latter is true, ORC may play the role of Rif2 at telomeres in yeast species that diverged from $S$. cerevisiae prior to the whole-genome duplication and, by extension, in other eukaryotes. Interestingly, ORC is recruited to telomeres by TRF2 in human cells (Deng et al. 2007). Whether ORC directly inhibits NHEJ, elongation by telomerase, and homologous recombination in some species remains to be tested.

\section{Materials and methods}

Strains and plasmids

The strains used in this study are listed in Supplemental Table 1. Silencing-defective strains are deleted of $H M L \alpha$ or $H M R \boldsymbol{a}$ to remain NHEJproficient unless mentioned. Details of strain construction are provided with the Supplemental Material.

Amplifications of the telomere-telomere fusions

Cells were grown to saturation in rich medium for $4 \mathrm{~d}$ (Fig. 1) or $3 \mathrm{~d}$ (Figs. 2,3 ). Telomere-telomere fusions were amplified by PCR from $\sim 10 \mathrm{ng}$ of genomic DNA (see Supplemental Material). The mean length of the fusions was obtained by subtracting the size of the amplified $\mathrm{X}$ and $\mathrm{Y}^{\prime}$ sequences $(460 \mathrm{bp}$ ) from the mean length of the amplified products.

Telomere length

Telomere length was measured by PCR after end-labeling with terminal transferase (Supplemental Material; Teixeira et al. 2004). The mean telo- 
mere length was obtained by subtracting the size of the end primer $(26$ $\mathrm{bp}$ ) and the size of the amplified $\mathrm{X}$ or $\mathrm{Y}^{\prime}$ sequences (340 or $120 \mathrm{bp}$, respectively) from the mean length of the amplified products.

I-SceI assay

Cells were spread at appropriate dilutions on glucose- and galactosecontaining plates (Supplemental Material). Colonies were counted after incubation for $4 \mathrm{~d}$ at $30^{\circ} \mathrm{C}$.

Alignment of the Rif2 and Orc4 sequences

A similarity between Rif2 and Kluyveromyces lactis Orc4 could be revealed by PSI-BLAST searches just below the threshold value when no compositional adjustment was preferred. This similarity was supported at the secondary structure level using Hydrophobic Cluster Analysis (HCA) (Supplemental Material; Supplemental Fig. 2).

\section{Acknowledgment}

We thank Cécile Fairhead, Marie-Claude Marsolier-Kergoat, Steve Buck, and David Shore for plasmids, and Emmanuelle Martini, Vincent Pennaneach, Miguel Godinho Ferreira, Francis Fabre, Serge Gangloff, and Karine Dubrana for suggestions, comments, and encouragements. This work is supported by grants from ARC, ANR (ANR-06-BLAN-0076), and INCa. A.G. was supported by a Fondation de France post-doctoral fellowship.

\section{References}

Amiard, S., Doudeau, M., Pinte, S., Poulet, A., Lenain, C., FaivreMoskalenko, C., Angelov, D., Hug, N., Vindigni, A., Bouvet, P., et al. 2007. A topological mechanism for TRF2-enhanced strand invasion. Nat. Struct. Mol. Biol. 14: 147-154.

Astrom, S.U., Okamura, S.M., and Rine, J. 1999. Yeast cell-type regulation of DNA repair. Nature 397: 310.

Bae, N.S. and Baumann, P. 2007. A RAP1/TRF2 complex inhibits nonhomologous end-joining at human telomeric DNA ends. Mol. Cell 26: $323-334$

Bowers, J.L., Randell, J.C., Chen, S., and Bell, S.P. 2004. ATP hydrolysis by ORC catalyzes reiterative $\mathrm{Mcm} 2-7$ assembly at a defined origin of replication. Mol. Cell 16: 967-978.

Buck, S.W. and Shore, D. 1995. Action of a RAP1 carboxy-terminal silencing domain reveals an underlying competition between HMR and telomeres in yeast. Genes \& Dev. 9: 370-384.

Celli, G.B. and de Lange, T. 2005. DNA processing is not required for ATM-mediated telomere damage response after TRF2 deletion. Nat. Cell Biol. 7: 712-718.

Celli, G.B., Denchi, E.L., and de Lange, T. 2006. Ku70 stimulates fusion of dysfunctional telomeres yet protects chromosome ends from homologous recombination. Nat. Cell Biol. 8: 885-890.

Chan, S.W. and Blackburn, E.H. 2003. Telomerase and ATM/Tellp protect telomeres from nonhomologous end joining. Mol. Cell 11: 13791387.

Craven, R.J. and Petes, T.D. 1999. Dependence of the regulation of telomere length on the type of subtelomeric repeat in the yeast Saccharomyces cerevisiae. Genetics 152: 1531-1541.

Deng, Z., Dheekollu, J., Broccoli, D., Dutta, A., and Lieberman, P.M. 2007. The origin recognition complex localizes to telomere repeats and prevents telomere-circle formation. Curr. Biol. 17: 1989-1995.

Dreesen, O., Li, B., and Cross, G.A. 2007. Telomere structure and function in trypanosomes: A proposal. Nat. Rev. Microbiol. 5: 70-75.

DuBois, M.L., Haimberger, Z.W., McIntosh, M.W., and Gottschling, D.E. 2002. A quantitative assay for telomere protection in Saccharomyces cerevisiae. Genetics 161: 995-1013.

Ferreira, M.G. and Cooper, J.P. 2001. The fission yeast Tazl protein protects chromosomes from Ku-dependent end-to-end fusions. Mol. Cell 7: $55-63$.

Griffith, J.D., Comeau, L., Rosenfield, S., Stansel, R.M., Bianchi, A., Moss, H., and de Lange, T. 1999. Mammalian telomeres end in a large duplex loop. Cell 97: 503-514.

Kyrion, G., Boakye, K.A., and Lustig, A.J. 1992. C-terminal truncation of RAP1 results in the deregulation of telomere size, stability, and function in Saccharomyces cerevisiae. Mol. Cell. Biol. 12: 5159-5173.
Kyrion, G., Liu, K., Liu, C., and Lustig, A.J. 1993. RAP1 and telomere structure regulate telomere position effects in Saccharomyces cerevisiae. Genes \& Dev. 7: 1146-1159.

Lee, K. and Lee, S.E. 2007. Saccharomyces cerevisiae Sae2- and Tel1dependent single strand DNA formation at DNA break promotes microhomology-mediated end joining. Genetics 173: 2003-2014.

Levy, D.L. and Blackburn, E.H. 2004. Counting of Rif1p and Rif2p on Saccharomyces cerevisiae telomeres regulates telomere length. Mol. Cell. Biol. 24: 10857-10867.

Li, B., Oestreich, S., and de Lange, T. 2000. Identification of human Rap1: Implications for telomere evolution. Cell 101: 471-483.

Liu, J., Smith, C.L., DeRyckere, D., DeAngelis, K., Martin, G.S., and Berger, J.M. 2000. Structure and function of Cdc6/Cdc18: Implications for origin recognition and checkpoint control. Mol. Cell 6: 637648.

Marcand, S., Gilson, E., and Shore, D. 1997. A protein-counting mechanism for telomere length regulation in yeast. Science 275: 986-990.

Mieczkowski, P.A., Mieczkowska, J.O., Dominska, M., and Petes, T.D. 2003. Genetic regulation of telomere-telomere fusions in the yeast Saccharomyces cerevisae. Proc. Nat1. Acad. Sci. 100: 10854-10859.

Miller, K.M., Ferreira, M.G., and Cooper, J.P. 2005. Taz1, Rap1 and Rif1 act both interdependently and independently to maintain telomeres. EMBO I. 24: 3128-3135.

Mishra, K. and Shore, D. 1999. Yeast Ku protein plays a direct role in telomeric silencing and counteracts inhibition by rif proteins. Curr. Biol. 9: 1123-1126.

Moretti, P., Freeman, K., Coodly, L., and Shore, D. 1994. Evidence that a complex of SIR proteins interacts with the silencer and telomerebinding protein RAP1. Genes \& Dev. 8: 2257-2269.

Negrini, S., Ribaud, V., Bianchi, A., and Shore, D. 2007. DNA breaks are masked by multiple Rapl binding in yeast: Implications for telomere capping and telomerase regulation. Genes \& Dev. 21: 292-302.

Pardo, B. and Marcand, S. 2005. Rap1 prevents telomere fusions by nonhomologous end joining. EMBO I. 24: 3117-3127.

Pardo, B., Ma, E., and Marcand, S. 2006. Mismatch tolerance by DNA polymerase Pol4 in the course of nonhomologous end joining in Saccharomyces cerevisiae. Genetics 172: 2689-2694.

Pennaneach, V. and Kolodner, R.D. 2004. Recombination and the Tell and Mecl checkpoints differentially effect genome rearrangements driven by telomere dysfunction in yeast. Nat. Genet. 36: 612-617.

Ribes-Zamora, A., Mihalek, I., Lichtarge, O., and Bertuch, A.A. 2007. Distinct faces of the Ku heterodimer mediate DNA repair and telomeric functions. Nat. Struct. Mol. Biol. 14: 301-307.

Riha, K., Heacock, M.L., and Shippen, D.E. 2006. The role of the nonhomologous end-joining DNA double-strand break repair pathway in telomere biology. Annu. Rev. Genet. 40: 237-277.

Rost, B., Yachdav, G., and Liu, J. 2004. The PredictProtein server. Nucleic Acids Res. 32: W321-W326. doi: 10.1093/nar/gkh377.

Scannell, D.R., Frank, A.C., Conant, G.C., Byrne, K.P., Woolfit, M., and Wolfe, K.H. 2007. Independent sorting-out of thousands of duplicated gene pairs in two yeast species descended from a whole-genome duplication. Proc. Natl. Acad. Sci. 104: 8397-8402.

Teixeira, M.T., Arneric, M., Sperisen, P., and Lingner, J. 2004. Telomere length homeostasis is achieved via a switch between telomeraseextendible and -nonextendible states. Cell 117: 323-335.

Teng, S.C., Chang, J., McCowan, B., and Zakian, V.A. 2000. Telomeraseindependent lengthening of yeast telomeres occurs by an abrupt Rad50p-dependent, Rif-inhibited recombinational process. Mol. Cell 6: $947-952$.

van Steensel, B., Smogorzewska, A., and de Lange, T. 1998. TRF2 protects human telomeres from end-to-end fusions. Cell 92: 401-413.

Viscardi, V., Bonetti, D., Cartagena-Lirola, H., Lucchini, G., and Longhese, M.P. 2007. MRX-dependent DNA damage response to short telomeres. Mol. Biol. Cell 18: 3047-3058.

Wotton, D. and Shore, D. 1997. A novel Raplp-interacting factor, Rif2p, cooperates with Riflp to regulate telomere length in Saccharomyces cerevisiae. Genes \& Dev. 11: 748-760. 


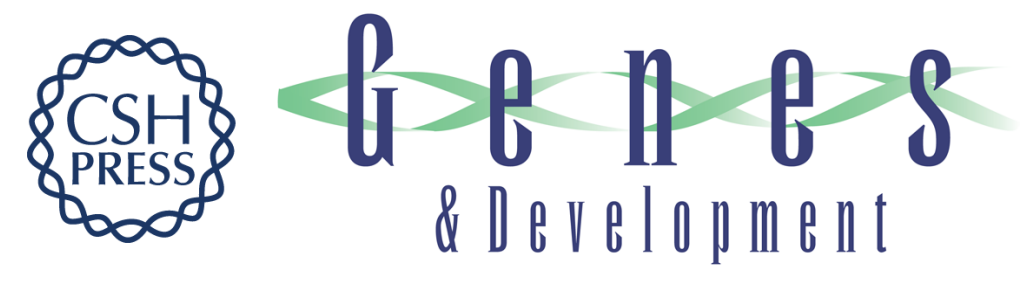

\section{Multiple pathways inhibit NHEJ at telomeres}

Stéphane Marcand, Benjamin Pardo, Ariane Gratias, et al.

Genes Dev. 2008, 22:

Access the most recent version at doi:10.1101/gad.455108

Supplemental

Material

References

This article cites 38 articles, 15 of which can be accessed free at:

http://genesdev.cshlp.org/content/22/9/1153.full.html\#ref-list-1

\section{License}

Email Alerting Service right corner of the article or click here.
http://genesdev.cshlp.org/content/suppl/2008/04/14/22.9.1153.DC1

Receive free email alerts when new articles cite this article - sign up in the box at the top

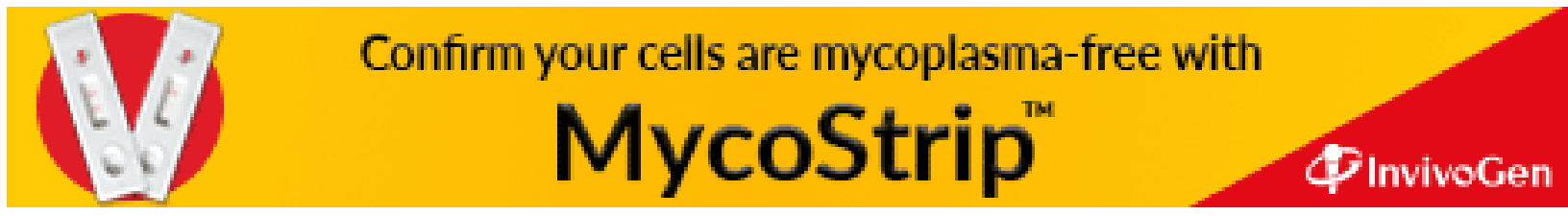

